\title{
The Effect of a Gravity Wave Drag Parameterization Scheme on GLA Fourth Order GCM Forecasts
}

\author{
By H.M. Helfand, J.C. Jusem ${ }^{1)}$, J. Pfaendtner ${ }^{1)}$, J. Tenenbaum ${ }^{2)}$ and E. Kalnay ${ }^{32}$ \\ Laboratory for Atmospheres, NASA/Goddard Space Flight Center, Greenbelt, MD 20771 U.S.A. \\ (Manuscript received 29 November 1986, in revised form 2 March 1987)
}

\begin{abstract}
In agreement with the experience of other centers, the high resolution GLA Fourth Order GCM has been found to exhibit a pronounced climate drift toward a state of westerly bias in the Northern Hemisphere which is not nearly so evident in the coarser resolution model. This climate drift causes the fine resolution model to lose its superiority in predictive skill over the coarser model after only about 5 days of a typical forecast.

Following Palmer et al. (1986), a simple gravity wave parameterization scheme has been implemented in the GLA model which has reduced the westerly bias in the Northern Hemisphere, has improved forecast skill by about 12 hours and has resulted in maintaining the superiority in forecast skill of the high resolution model for at least 10 days.

The error reduction due to the gravity waves is immediately apparent in the stratosphere, where the improvement is confined mainly to the zonal mean component although spurious jet streaks in the vicinity of mountainous regions are also removed. Error reduction occurs in the middle and lower troposphere only after about 5 days and is associated with a secondary meridional circulation generated in response to the gravity wave drag in the stratosphere.

Improvements are observed in the Northern Hemisphere climatology where low level westerlies are weakened and shifted poleward and, unexpectedly, in the Southern Hemisphere where the roaring forties and fifties are considerably better simulated.

The positive impact on forecast skill and climate simulation of the simple gravity wave scheme has provided motivation for the development of a more advanced scheme, and the plans for such development are outlined.
\end{abstract}

\section{Introduction}

When the horizontal resolution of the Goddard Laboratory for Atmospheres (GLA) Fourth Order General Circulation Model (GCM) was doubled from $4^{\circ}$ latitude by $5^{\circ}$ longtitude to $2^{\circ}$ latitude by $2.5^{\circ}$ longitude, the forecast skill of the model was improved and the model's systematic errors were reduced for the first 5 days of the integration (Helfand et al., 1987). However, beyond day 7, the

1) Sigma Data Services Corporation.

2) Division of Natural Sciences, SUNY, Purchase, NY 10577.

3) Present affiliation: National Meteorological Center, Washington, D.C. systematic errors of the fine resolution model exceeded those of the coarse model with the largest error in the zonal mean component. The medium-range systematic errors for the fine resolution model in the wintertime Northern Hemisphere correspond to sea level pressure and mean westerly wind biases also found in the model's winter climatology.

The wintertime climate biases of the highresolution GLA model resemble those experienced with the Canadian Climate Centre (CCC) Spectral Atmospheric GCM (Boer et al., 1984), the medium-resolution version of the British Meteorological Office 11-Layer AGCM (Slingo and Pearson, 1986) and with other 
models listed in Helfand et al. (1987). Recently, it has been demonstrated that simple, orographic gravity-wave-drag parameterization schemes can alleviate these biases in the CCC model (Boer et al., 1984; McFarlane, 1987) and the British Meteorological Office model (Palmer et al., 1986). Such schemes have also resulted in reduction of systematic errors in the Recherche en Prévision Numerique (RPN) Spectral Model (Chouinard et al., 1986) and in the British Meteorological Office 15-Layer Operational Model (Kitchen and Dickinson, 1987).

A simple orographic gravity wave drag scheme similar to those of McFarlane (1987) and Palmer et al. (1986) has been introduced into the GLA Fourth Order GCM to test whether it would reduce the climate drift of the fine resolution model and improve the model's medium range predictive skill. In this paper, we present the results of a small number of ten-day forecast experiments carried out both with and without the gravity wave scheme at the $2^{\circ} \times 2.5^{\circ}$ resolution. The GLA model is described by Helfand et al. (1987) elsewhere in this volume and in greater detail by Kalnay et al. (1983).

In section 2, we describe the orographic gravity wave parameterization scheme we have used in this study. Section 3 presents the horizontal and vertical distribution of the gravity wave forcing term in the integrations. The impact of the gravity wave drag on the GLA model's predictive skill, on its systematic errors and on its climatology are discussed in section 4 . Section 5 contains a summary and an outline of future plans.

\section{Description of the gravity wave parameterization scheme}

Because the intent of this study is to investigate the impact of gravity wave drag on the predictive skill of the GLA Fourth Order Moder Model, the parameterization scheme that has been chosen to represent gravity waves is a fairly simple one. The scheme is similar to the one developed by Palmer et al. (1986) (hereafter denoted by PSS) for the British Meteorological Office model. In this steady-state, linear scheme, as in that of
PSS, it is assumed that sub-grid-scale fluctuations of orographic height generate sub-gridscale, vertically propagating gravity waves in the hydrostatic, nonrotating range and that the grid-box averaged momentum flux due to these waves can be taken to be

$$
(\overline{\rho \boldsymbol{u} w})=\rho \boldsymbol{U} N k \sigma_{h}^{2}
$$

at the earth's surface (PSS, Eq. 3.1), where $\rho, U$ and $N$ are the density, wind vector and Brunt-Väisälä frequency of the atmosphere at the $\sigma=.889$ level, $k$ is a tunable parameter which represents in some sense the effective wave number for gravity waves in the direction of the surface wind and $\sigma_{\hbar}^{2}$ is the subgrid-scale variance of the orography as computed from the U.S. Navy 10 minute topographic data set. Following PSS, we have prescribed $k=2.5 \times 10^{-5} \mathrm{~m}^{-1}$, although if one interprets $k$ as the wave number for a monochromatic spectrum, this corresponds to a wavelength of more than $200 \mathrm{~km}$ for which rotational effects are not negligible.

In the current application of (1), the subgrid-scale orographic variance $\sigma_{h}^{2}$ has been limited to a maximum value of $(400 \mathrm{~m})^{2}$ as in PSS. PSS adopted this upper bound to avoid numerical instabilities, but it can be justified by a quasi-geostrophic scale analysis in which one insists that the gravity wave forcing be smaller than the Coriolis force at the level of of maximum stress divergence (see the Appendix).

PSS take the amplitude $h$ of the groundlevel deflection due to the gravity wave to be $h=\sigma_{h}$ so that the $(400 \mathrm{~m})^{2}$ limitation on the orographic variance corresponds to a $400 \mathrm{~m}$ limit on the surface value of $h$. The current scheme imposes an additional limitation on the surface value of $h$. Miles and Huppert (1969) found with a steady-state, nonlinear gravity wave model and Peltier and Clark (1983) confirmed with a nonlinear, timedependent model that nonlinear effects become critical and that gravity waves do not propagate upward through the tropopause as assumed by linear theory when the condition

$$
F r \equiv \frac{N h}{U}<.85
$$


is violated at the ground. Here $F r$ is the Froude number. Thus the current scheme limits the surface deflection due to vertically propagating gravity waves by

$$
h \leqq .85 U / N \text {. }
$$

This is done by reducing the surface momentum flux (1) by the factor $(.85 / F r)^{2}$ wherever (2) is violated since the momentum flux is proportional to $h^{2}$. PSS have run some experiments with a condition similar to (3) but with .85 replaced by unity.

Eliassen and Palm (1961) have shown that in the absence of turbulent dissipation or a critical level, the upward flux of momentum does not change with height. Thus in the current scheme, as in the scheme of PSS, the vertical momentum flux of the gravity waves $\overline{\rho \boldsymbol{u} w}$ does not decrease with heightunt il one of the following conditions occurs : a critical level

$$
U \cdot U_{\text {surface }}=0
$$

is encountered, at which point the momentum flux vanishes; the gravity waves break and generate turbulence; or the top of the model is reached.

Breaking occurs in the free atmosphere in our model when the local value of the wavemodified Richardson number becomes less than or equal to the critical Richardson number $R i_{c}=.25$ at the phase of the (monochromatic) wave for which the decrease in the local static stability is maximized. The local wavemodified Richardson number $R i_{m}$ at that point is

$$
R i_{m}=R i\left(1-\frac{N h}{U}\right),
$$

where $R i$ is the Richardson number for the mean state of the atmosphere. In contrast, PSS compute a local Richardson number in which the maximum wave-induced decrease in local stability and the maximum waveinduced increase in the local wind shear are assumed to be in phase, even though they are actually out of phase by $\pi / 2$, and in which the local shear normal to the direction of the wave is increased as well as the local shear in the direction of the wave, even though the normal shear is not actually af- fected by the wave. Thus, they obtain their Eq. (10) instead of (5). In our model, the wave breaking occurs at the level where the amplification of the wave causes the local Froude number to exceed the critical value

$$
F r_{c}=\frac{N h_{c}}{U}=1-\frac{R i_{c}}{R i}=1-\frac{1}{4 R i},
$$

in contrast to PSS's Eq. (11) for their critical local Froude number.

Once the gravity wave begins to break, both in the present model and in the model of PSS, the wave saturation hypothesis of Lindzen (1981) is invoked, in which it is assumed that the wave-induced turbulent dissipation prevents the displacement amplitude $h$ from exceeding its critical value $h_{c}$. That is, if the value computed for $F r$ at a given level is found to exceed the critical value $F r_{c}$ for that level, it is assumed that the turbulence generated by the breaking of the wave will reduce the wave momentum flux by the factor $\left(F r_{c} / F r\right)^{2}$.

The current GLA tropospheric model does not resolve the stratosphere well, having only one layer between $110 \mathrm{mb}$ and the top of the model at $10 \mathrm{mb}$. Presently we assume that all of the momentum flux associated with the gravity waves which reaches the top layer is deposited there so that $\overline{\rho \boldsymbol{u} w}$ vanishes at the $10 \mathrm{mb}$ level.

After the vertical profile of the momentum flux has been determined for a model column, the gravity wave drag is computed as the vertical divergence of the flux.

\section{Distribution of gravity wave forcing}

To assess the effect of the gravity wave drag on forecast skill, a series of three 10day forecasts was run at the $2^{\circ}$ latitude by $2.5^{\circ}$ longitude resolution both with and without the gravity wave parameterization scheme described in 2. Initial conditions were obtained from a $4^{\circ} \times 5^{\circ}$ GLA analysis of the first FGGE special observing period (SOP-I) using a model which did not include the gravity wave scheme. Forecasts were started from 12 January, 1 February and 15 February 1979 initial conditions and were run with an enhanced "significant heights" orography 
(Pfaendtner et al., 1985). In addition, $4^{\circ} \times 5^{\circ}$ forecasts were run from these dates as well as from others with and without the gravity wave parameterization.

Fig. 1 shows a typical 10-day average of the vector components and of the magnitude of the surface stress generated by the orographic gravity wave parameterization scheme in the $2^{\circ} \times 2.5^{\circ}$ model. Surface stresses in the other $2^{\circ} \times 2.5^{\circ}$ and $4^{\circ} \times 5^{\circ}$ integrations were rather similar. The distribution of stresses generally reflects the presence of orography with some significant differences. For example, the largest values are found in the Pyrenees and in central and eastern Asia rather than in the Tibetan Plateau where the ground height reaches its maximum. This is due to differences in stability and wind speed at lower levels and to the artificial bound of $(400 \mathrm{~m})^{2}$ imposed upon the topographic variance. Also, higher terrain is not necesarily rougher as is indicated by a simple inspection of the
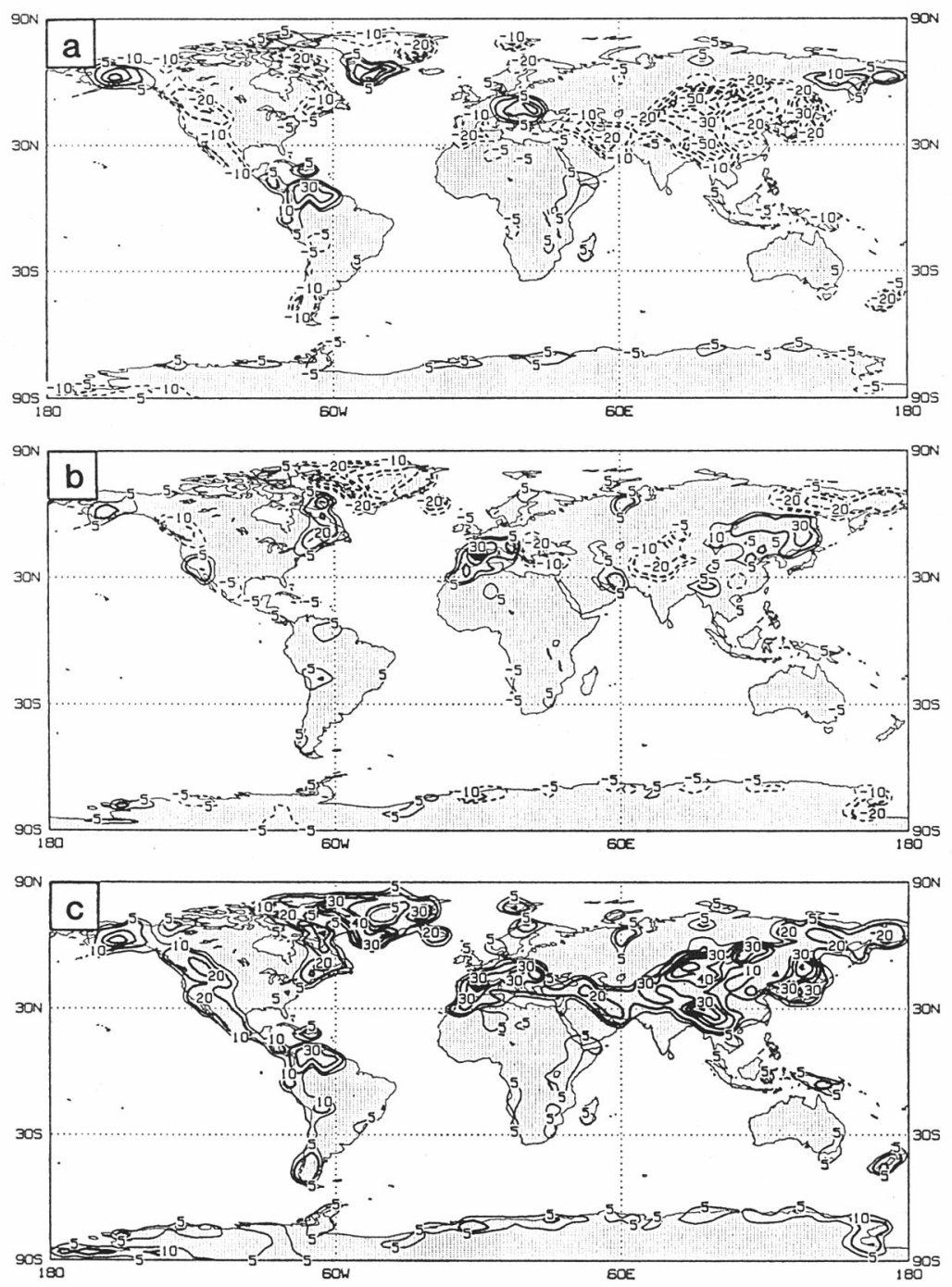

Fig. 1 Ten-day average of the orographic gravity wave surface stress for the case initialized from 0000 GMT 15 February 1979 . (a) Zonal component. (b) Meridional component. (c) Average magnitude of the stress. The light solid and dashed contours represent $\pm 5 \times 10^{-2} \mathrm{Nm}^{-2}$, respectively, while the heavy solid and dashed contours represent \pm 10 $\times 10^{-2} \mathrm{Nm}^{-2}, \pm 20 \times 10^{-2} \mathrm{Nm}^{-2}$, etc. 
stress pattern over central Greenland. In fact, gravity wave stress is enhanced along the edges of the Greenland Plateau by the strong contribution of the slope to the sub-grid-scale topographical variance. However, the peak values of 0.2 to $0.5 \mathrm{Nm}^{-2}$ are realistic and comparable with those obtained by PSS.

Fig. 2 shows the 10-day average of the acceleration by orographic gravity waves of the zonal component of the zonally averaged wind. There is a rather strong westward acceleration of up to $2-3 \times 10^{-5} \mathrm{~ms}^{-2}$ in the stratosphere between $30^{\circ} \mathrm{N}$ and $60^{\circ} \mathrm{N}$, and smaller values are also observed near the South Pole. A weaker eastward acceleration with with peak tropospheric values of about $5 \times 10^{-6} \mathrm{~ms}^{-2}$ takes place at latitudes poleward of $60^{\circ} \mathrm{N}$, in the tropics of the Northern Hemisphere and in a region centered at about $70^{\circ} \mathrm{S}$. The eastward acceleration in this latter region may be due in large part to the contribution of the topographic slope at the edge of the Antarctic continent to the production of orographic gravity waves.

The acceleration due to the gravity waves is at least an order of magnitude smaller than the Coriolis acceleration of the mean zonal flow. The regions of large westward acceleration in Fig. 2 occur above the jet and are due to the breaking of upward propagating gravity waves in regions of strong shear. In the regions of surface easterlies, the eastward acceleration due to gravity waves has a tropospheric peak near a critical level where

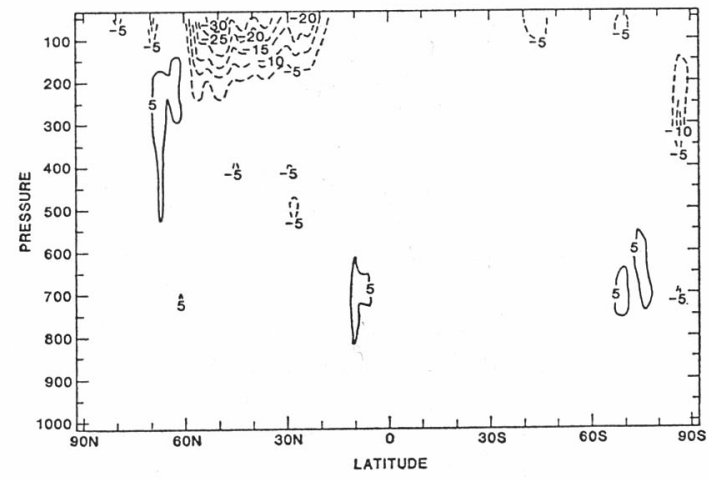

Fig. 2 Ten-day average of the acceleration of the mean zonal wind due to orographic gravity waves for the same case as in Fig. 1 Units are $10^{-6} \mathrm{~ms}^{-2}$ the wind direction changes to westerly.

As anticipated by Smith (1979) and shown in detail by PSS, the quasigeostrophic response to the forcing of Fig. 2 is a direct meridional circulation with a descending branch in the high latitudes of the Northern Hemisphere and an ascending branch over the tropics. The effect of this response is apparent very early in the forecast: after only 6 hours into the forecast (figure not shown) the polar stratosphere is more than one degree warmer in the run with gravity wave drag than in the control. The response to the subsidence poleward of $60^{\circ} \mathrm{N}$ is strongest in the stratosphere because of the large static stability.

\section{The impact of gravity wave drag on model predictions}

Although at this time we have made only three 10-day forecasts with the gravity wave parameterization, they give us a good indication of how much impact the present gravity wave scheme will have and what kinds of changes can be expected. In examining the results for this 3-case ensemble, it will be helpful to look at changes in the ensemble statistics for forecast skill, the changes that occur in the upper level winds, where the drag is the strongest, and the changes in the composite error structure for the ensemble. In addition, a 31-day integration is investigated to give a preliminary indication of the effect of gravity wave drag on the $2^{\circ} \times 2.5^{\circ}$ model's climatology.

\subsection{Changes in forecast skill}

Ensemble mean anomaly correlations and RMS errors of $500 \mathrm{mb}$ heights verified against GLA analysis show no systematic effect of gravity wave drag for the first 5 days of forecasts for the Northern Hemisphere extratropics (Fig. 3). By day 6, however, the gravity wave parameterization adds about 12 hours of skill to the anomaly correlations and even more to the RMS error statistics. Over Europe (Fig. 4), the reduction in RMS errors is apparent by day 2, and the improvement both in anomaly correlations and in RMS errors is quite large after day 6. On the 

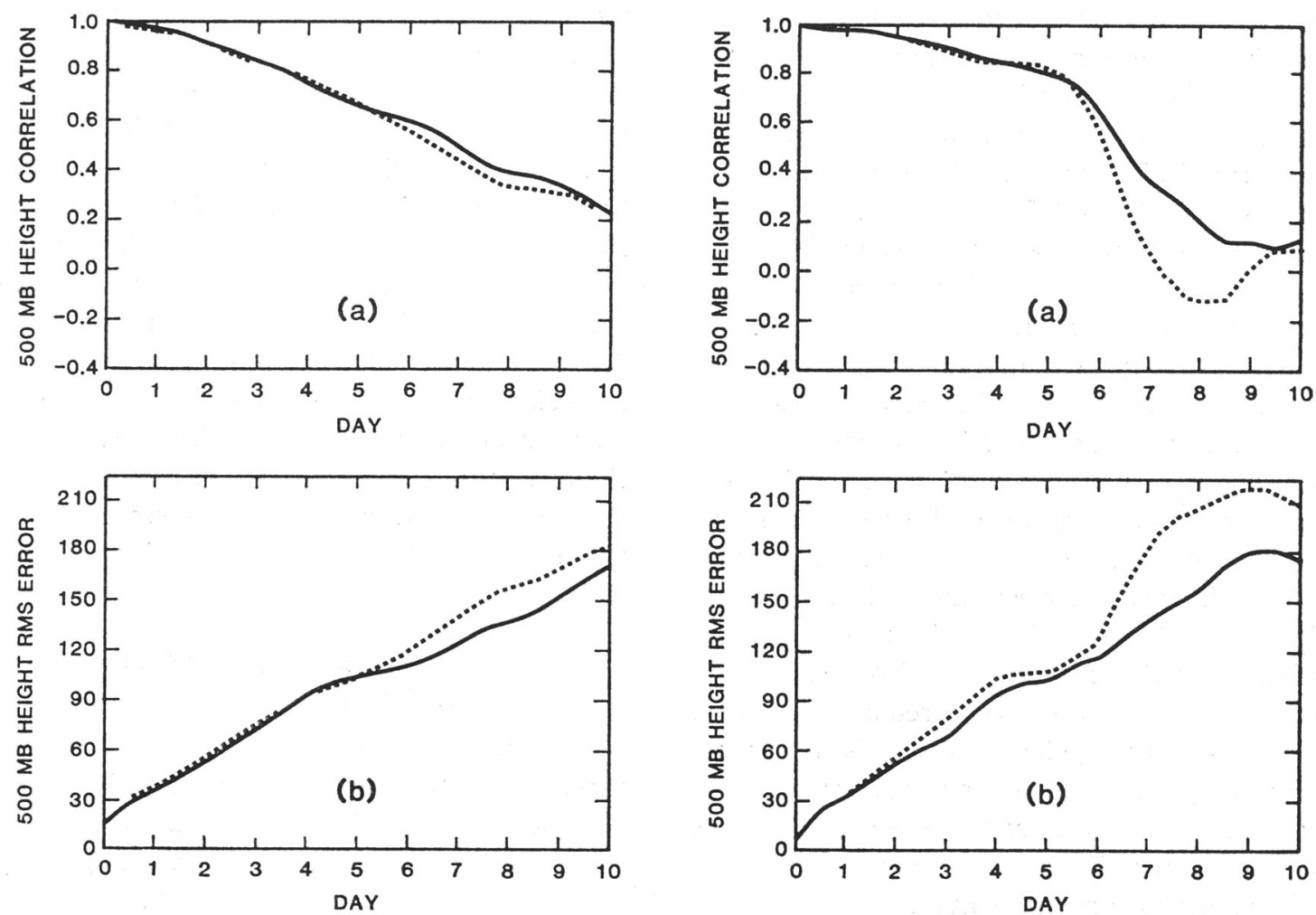

Fig. 3 Ensemble mean forecast statistics for 500 mb geopotential heights over the extratropics of the Northern Hemisphere verified against GLA analysis. Solid line for forecasts using the gravity wave parameterization, dashed line for forecasts without the parameterization (a) Anomaly correlation coefficient. (b) RMS error in meters.

other hand, ensemble statistics for the $4^{\circ} \times 5^{\circ}$ model (not shown) indicate that gravity wave drag diminishes forecast skill by about 12 hours from day 4 onward.

The impact of the gravity wave parameterization scheme can for some cases be much more significant than is indicated by the ensemble mean statistics of Fig. 3. The forecast from 12 January 1979 was the best prediction of the $2^{\circ} \times 2.5^{\circ}$ ensemble both when gravity wave drag was included and when it was not. Fig. 5 compares the $500 \mathrm{mb}$ anomaly correlations obtained using the coarser-resolution $4^{\circ} \times 5^{\circ}$ model with those obtained with the high-resolution model with and without the gravity wave parameterization. The increased resolution alone has extended the useful predictive skill of the GLA model by

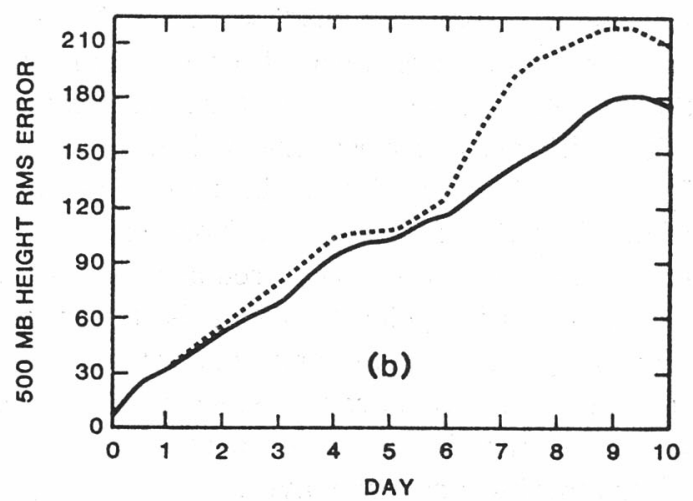

Fig. 4 As in Fig. 3, except for Europe.

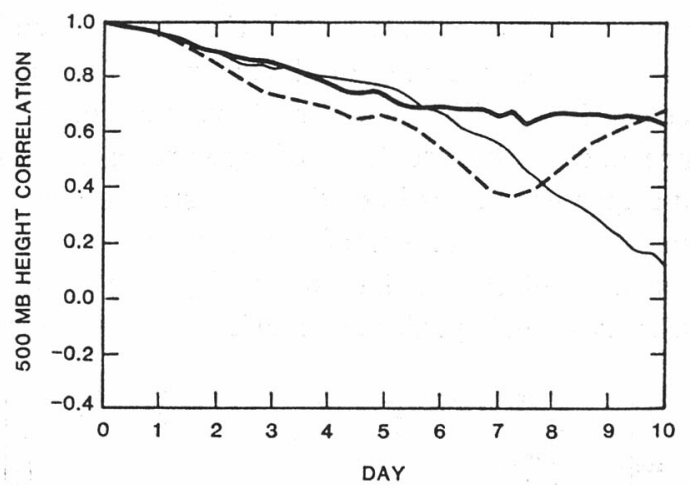

Fig. 5. Anomaly correlation coefficients for 500 mb geopotential heights over the extratropics of the Northern Hemisphere verified against GLA analysis for forecasts from 0000 GMT 12 January 1979. Heavy line for a forecast with the $2^{\circ} \times 2.5^{\circ}$ model including the gravity wave parameterization, solid line for a forecast with the $2^{\circ} \times 2.5^{\circ}$ model but not including the parameterization, dashed line for a forecast with the $4^{\circ} \times 5^{\circ}$ model without the parameterization.

about a day. Increased resolution plus the gravity wave drag scheme has extended the 
useful skill by more than four days. As was true for the ensemble statistics, the impact of gravity wave drag is not apparent at 500 $\mathrm{mb}$ unitl after day 5 . Unlike the ensemble, however, both $2^{\circ} \times 2.5^{\circ}$ forecasts are still rather skillful after 6 days probably due to the persistence of a blocking-high pattern over Eurasia (Helfand et al., 1987). At that time, the gravity wave impact contributes to the maintenance of forecast skill.

The impact of gravity wave drag is not felt simultaneously at all levels of the model. The effect of the gravity waves on $1000 \mathrm{mb}$ height error variance for the Northern Hemisphere (Fig. 6) is not seen until six days into the forecast. Although a significant portion of the improvement occurs in the zonal mean component, it should be noted that there is also a strong reduction of error in the zonally asymmetric flow. Impact at $500 \mathrm{mb}$ (Fig. 7) appears about a day earlier (in agreement with Fig. 3) and again the improvements are large in the zonal mean component. However, at the $100 \mathrm{mb}$ level (Fig. 8), the effect of the drag occurs almost immediately, as one might have expected from looking at the vertical distribution of the forcing due to gravity waves shown in Fig. 2. The improvements at this level, which for the first 8 days are entirely in the zonal mean component, are

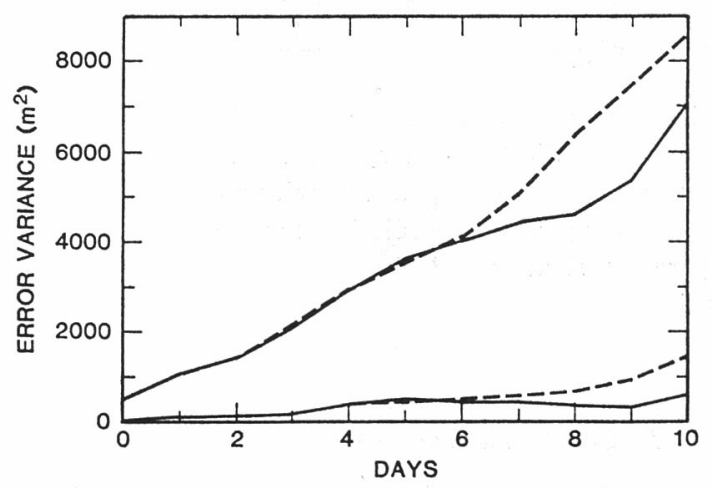

Fig. 6 Ensemble mean of $1000 \mathrm{mb}$ geopotential height error variance over the Northern Hemisphere. Solid lines for forecasts with the gravity wave parameterization, dashed lines for forecasts without the parameterization. Top curves are the total error variance, bottom curves are the part of the error variance due to zonal components $(m=0)$.

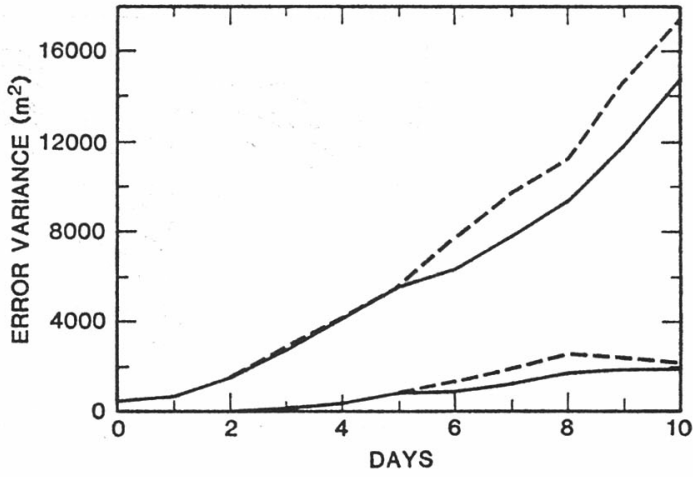

Fig. 7 As in Fig. 6, except for $500 \mathrm{mb}$ error variances.

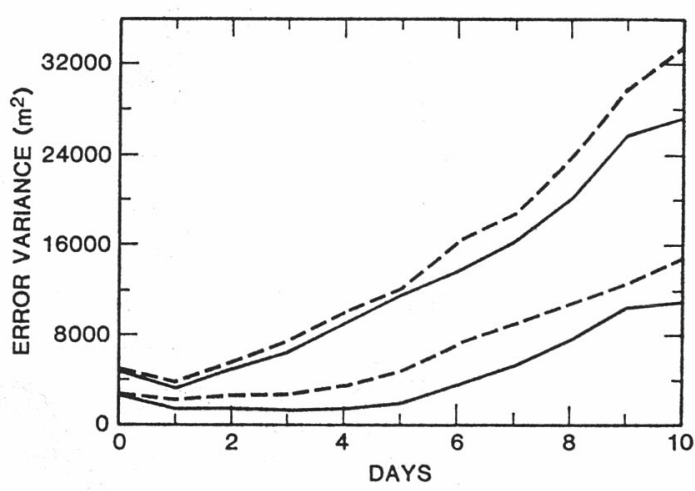

Fig. 8 As in Fig. 6, except for $100 \mathrm{mb}$ error variances.

due both to the direct reduction of a $100 \mathrm{mb}$ mean westerly wind bias by the wave drag itself and to the effects of a secondary Hadleytype circulation which is driven by the wave drag term as discussed in section 3 and in PSS. Such a secondary circulation in response to the wave drag forcing also helps to explain the delayed error reduction at the 500 $\mathrm{mb}$ and $1000 \mathrm{mb}$ levels.

Fig. 9 shows the reduction of the upper level winds due to the inclusion of the gravity wave drag scheme in the model in an 8-day forecast. Extraneous stratospheric wind streaks, which Tenenbaum $(1982,1983)$ has found to be typical in GLA Fourth Order Model and other model forecasts, have been removed east of the Urals and poleward of $60^{\circ} \mathrm{N}$. Qualitative disagreement with the analysis still remains in the region south of the Himalayas. It is possible that a more advanced gravity wave scheme might alleviate 

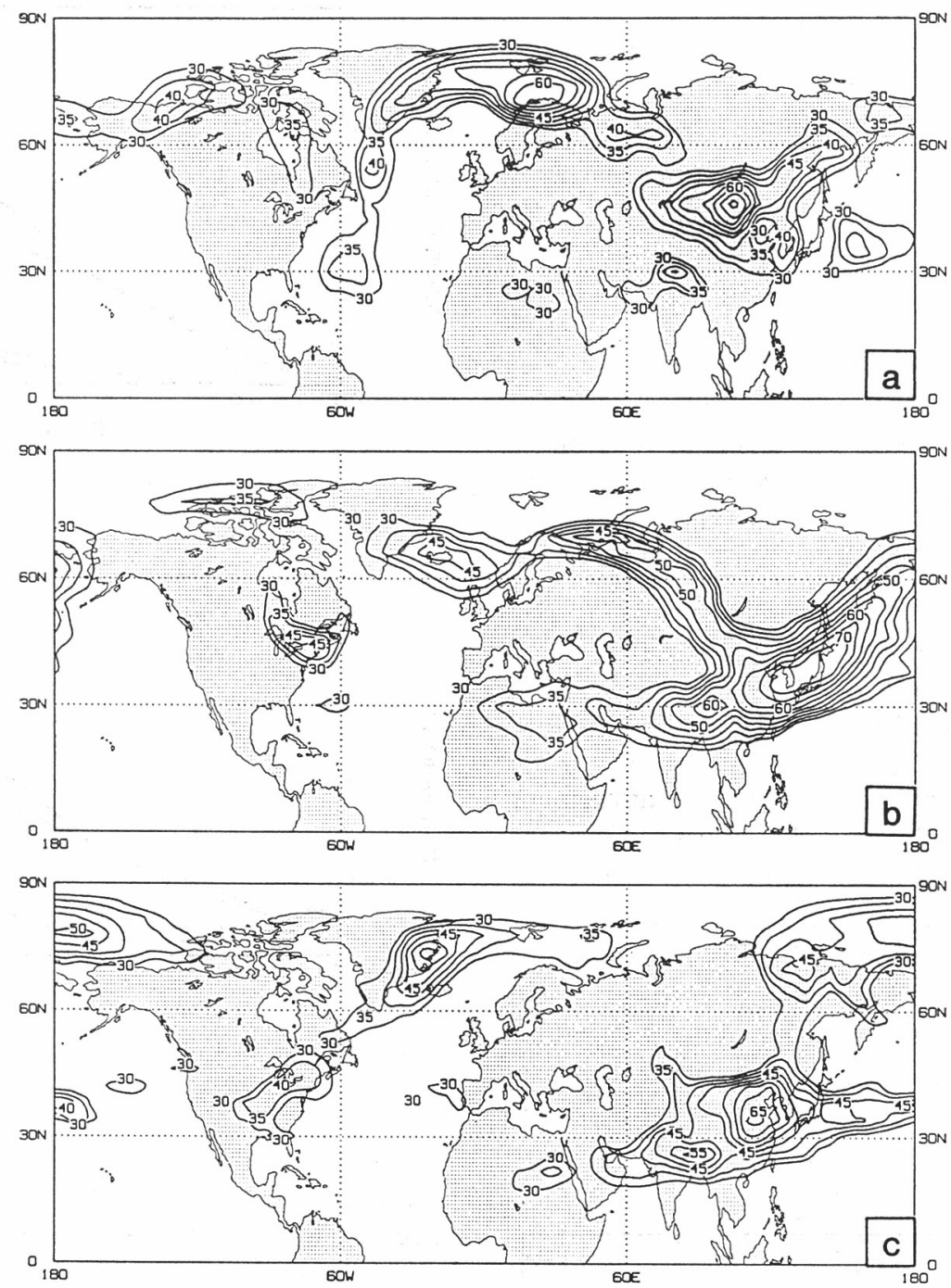

Fig. 9 Horizontal section of wind magnitude at the stratospheric level (10 $\mathrm{mb}$ to $120 \mathrm{mb}$ ). (a) GLA analysis for 0000 GMT 20 January 1979 . (b) 8-day model prediction for the same time by the GLA model without the gravity wave parameterization. (c) 8-day model prediction for the same time with the gravity wave parameterization. Units are $\mathrm{ms}^{-1}$. Isotachs below $30 \mathrm{~ms}^{-1}$ are suppressed.

this problem, especially if it took into consideration the pronounced anisotropy of the orography in this region and the possible generation of gravity waves by the strong shear of the subtropical jet. The effect of the gravity wave scheme on stratospheric winds is similar in most of the other $2^{\circ} \times 2.5^{\circ}$ and $4^{\circ} \times 5^{\circ}$ forecasts (see Tenenbaum, 1987, for another example).

\subsection{Composite forecast error}

Composite sea level pressure errors for day 4 of the 3 forecasts without the gravity wave parameterization scheme (the controls) and for the 3 forecasts with the scheme and their differences are shown in Figs. 10a, b, c. In large part, the error field has an equivalent barotropic structure, and the composite errors at $500 \mathrm{mb}$ are similar to those at sea level. 

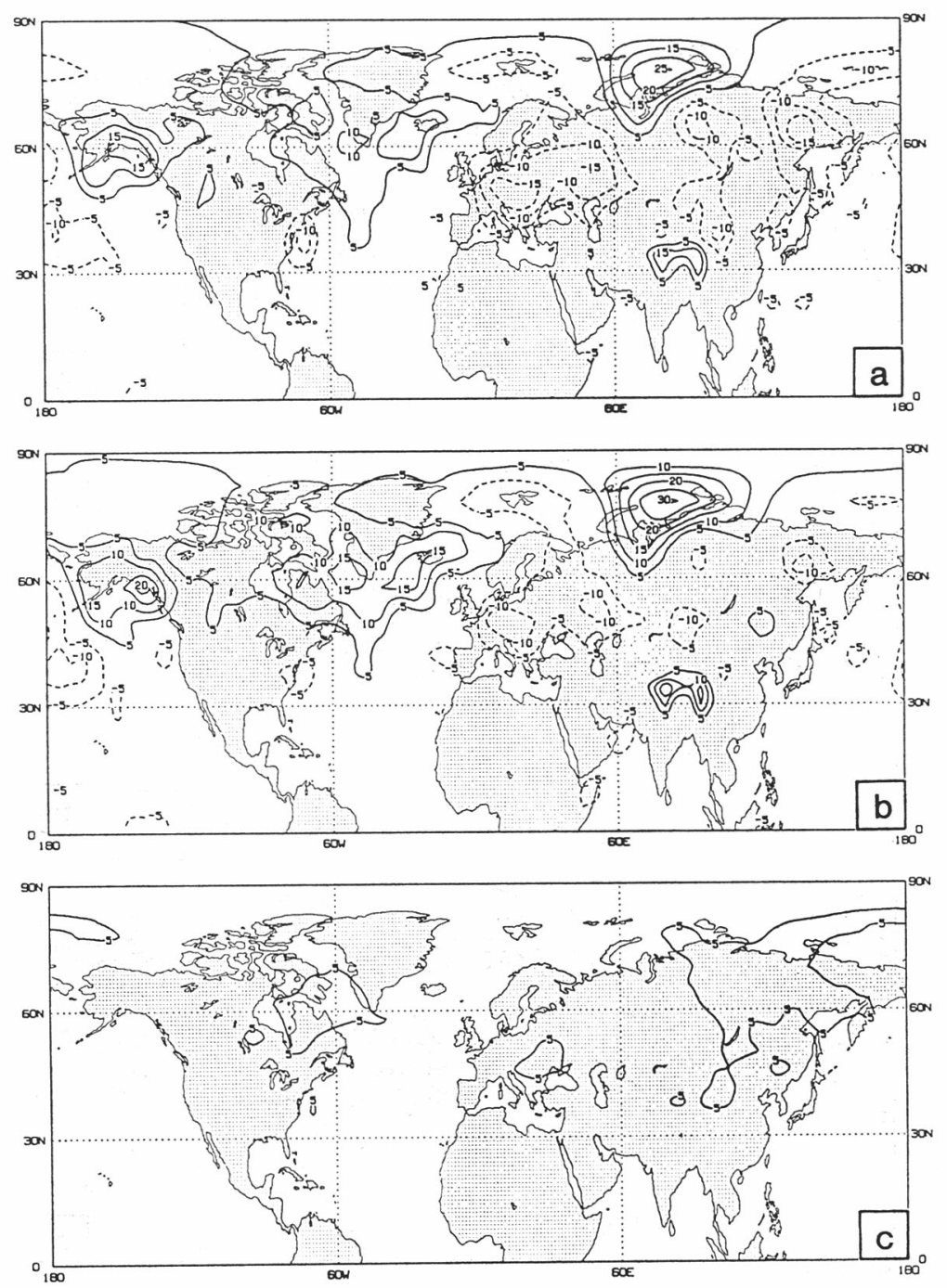

Fig. 10 (a) Composite sea level pressure errors for day 4 of the forecasts without the gravity wave parameterization. (b) The same for the forecasts with the gravity wave parameterization. (c) the difference between (b) and (a). Units are mb.

The composite errors for the control are similar to the 4-day systematic wintertime errors of the $2^{\circ} \times 2.5^{\circ}$ model (6 cases) presented by Helfand et al. (1987). There are large lows over Europe and central to eastern Asia, a small low off the east coast of the United States and highs over eastern Canada and Greenland, the region surrounding the Aleutian Islands and northernmost Asia. The gravity wave drag has resulted in a small reduction of the negative errors through suppression of the excessive mass transfer from the high latitudes to the low latitudes, as can be seen in Fig. 10c, and at the same time has allowed the positive errors to become somewhat larger.

After 8 days (Figs. 11a, b, c), the effects are much stronger. The meridional transfer of mass due to gravity wave drag is very apparent in Fig. 11c. The zonal character of the error field for the controls is no longer evident for the runs with the gravity wave scheme. The negative composite error of $36.5 \mathrm{mb}$ over Europe has been reduced to about 

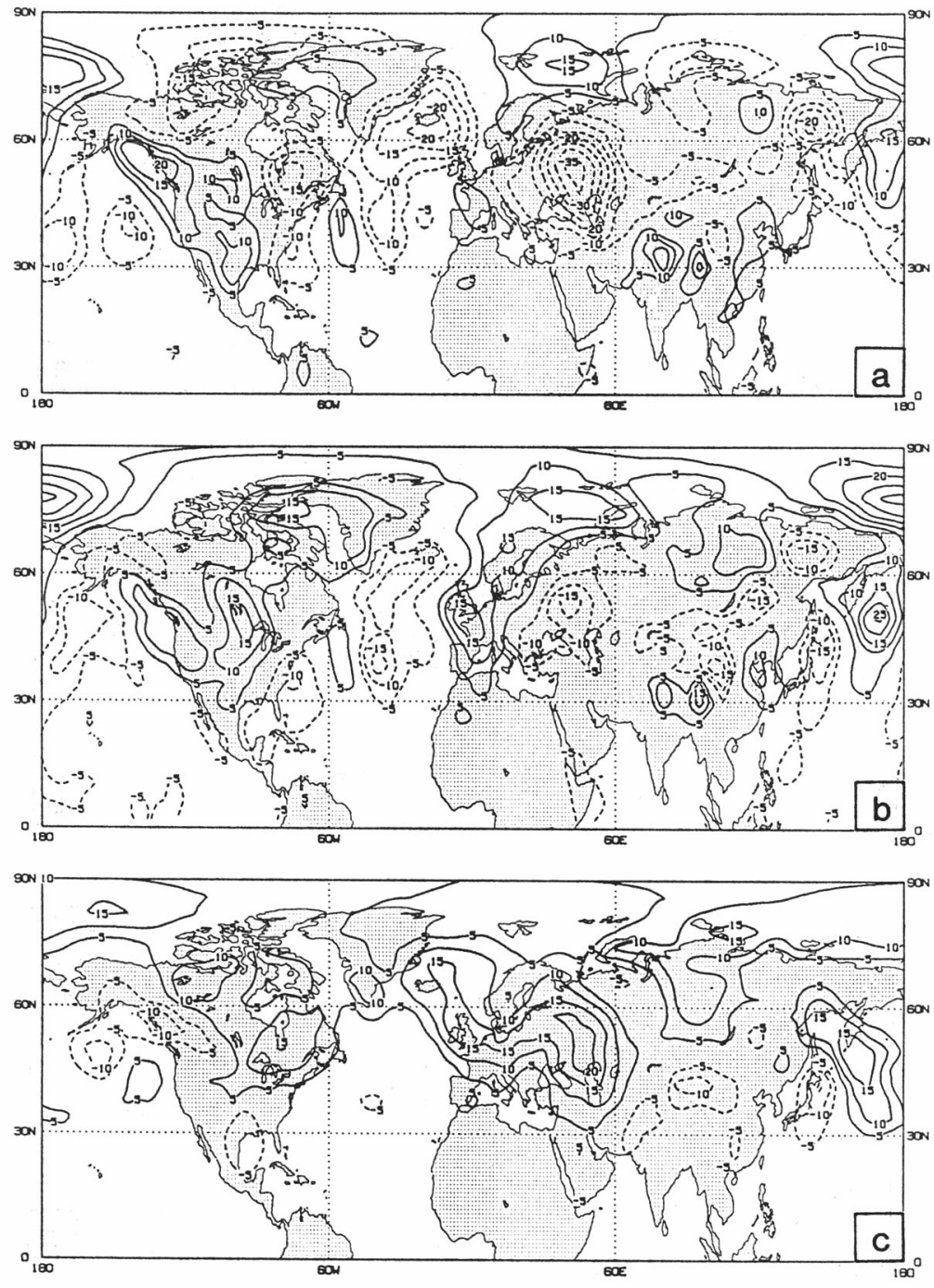

Fig. 11 The same as Fig. 10 but for day 8 of the forecasts.

half that value, and negative errors over the North Atlantic, the east coast of North America, northwestern Canada, the eastern Pacific and central Asia have also been significantly reduced. On the other hand, positive errors over western Europe and Scandinavia, Greenland and northeastern Canada, the Great Plains of North America and the region to the north and the east of Kamchatka have worsened.

Comparison of Fig. 11a with the wintertim 8-day systematic errors of the $2^{\circ} \times 2.5^{\circ}$ model ( 6 cases) indicates that some of the details of the error field might differ somewhat with a larger sample size but that the overall pattern will not.

\subsection{Effect on climate drift}

Extensive model integrations have not yet been performed with the new parameterization scheme. However, preliminary computations indicate that the gravity wave drag results in large changes, many of them good. Figs. 12 and 13 present time averages of sea level pressure for the last two weeks of 31 day integrations for January 1979 which were run with and without the gravity wave scheme. Although a single case does not represent the model climatology and some of the differences between these two runs are 


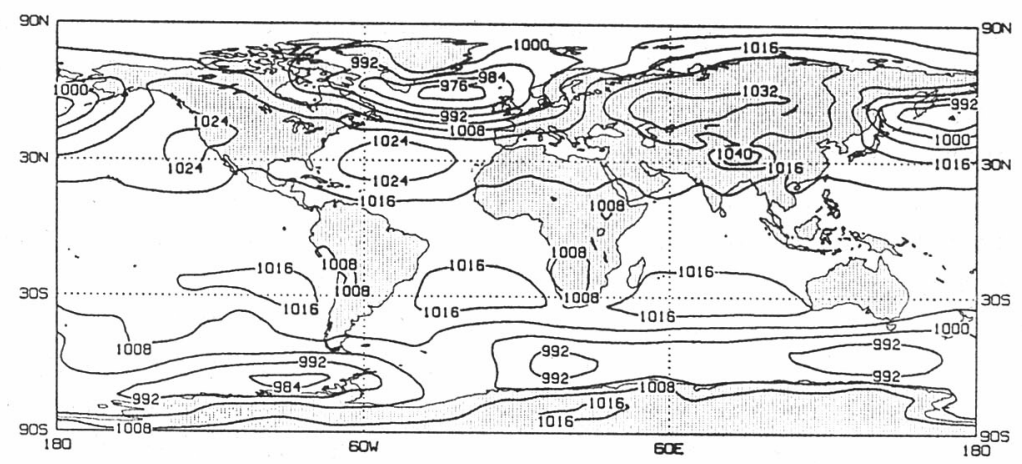

Fig. 12 Average sea level pressure for the last two weeks of a 31-day integration initialized from 0000 GMT 1 January 1979 which did not include the gravity wave parameterization.

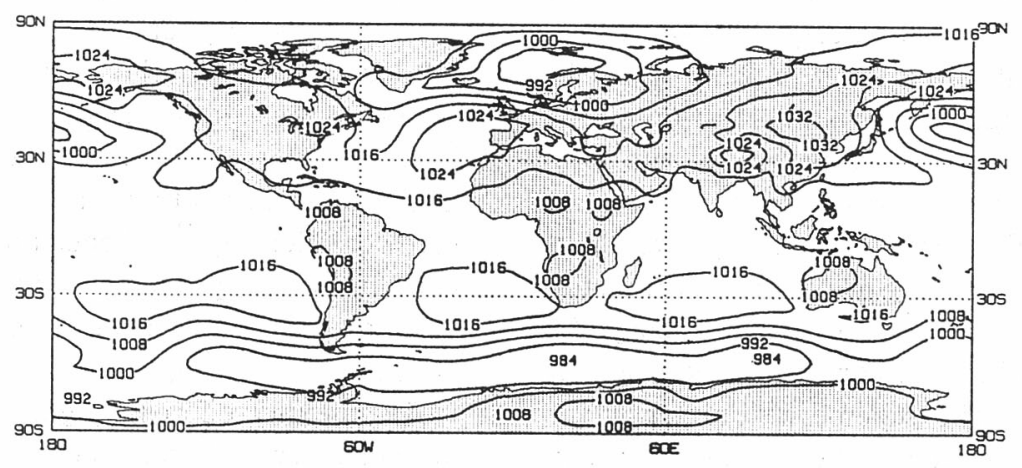

Fig. 13 The same as Fig. 12 but for an integration which included the gravity wave parameterization.

associated with natural variability, it is clear that the spuriously strong westerly flow between eastern Canada and western Europe in the control run has been greatly reduced in the integration with the gravity wave scheme

Unexpectedly, the circulation of the Southern Hemisphere has been significantly improved with a much better depiction of the roaring forties and the belt of low pressure at $60^{\circ} \mathrm{S}$ in the run with gravity wave drag. This improvement is probably due to the eastward acceleration by gravity waves which occurs near the edge of the Antarctic continent (see Figs. 1 and 2). This acceleration occurs near the $600-700 \mathrm{mb}$ critical level over the Antarctic shelf where the surface easterlies vanish and change sign. (Fig. 14). The acceleration produces a net transfer of mass from high to low latitudes and results not only in a southward shift of the lowpressure belt around Antarctica, but also in

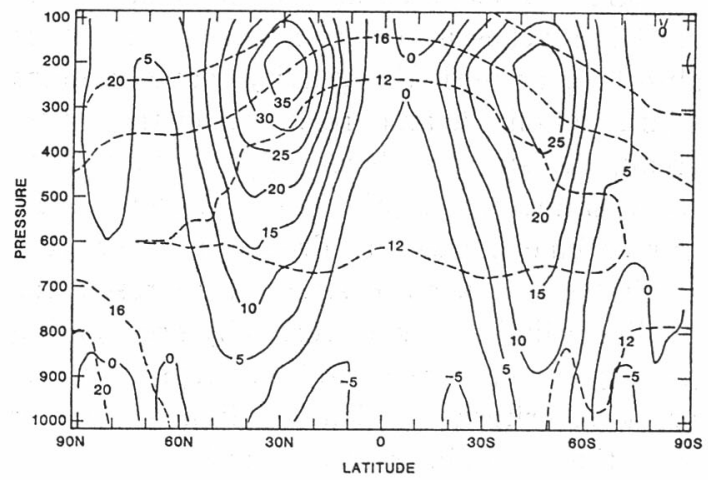

Fig. 14 Ten-day average of the zonally-averaged zonal wind in units of $\mathrm{ms}^{-1}$ (solid lines) and Brunt-Väisälä frequency $\mathrm{N}$ in units of $10^{-2} \mathrm{~s}^{-1}$ (dashed lines) for the same case as in Fig. 1.

more intense subtropical highs over the oceans, both in better agreement with observations.

\section{Summary and future plans}

In agreement with the experience of other 
centers, it has been observed by Helfand et al. (1987) that the high resolution $\left(2^{\circ}\right.$ lat by $2.5^{\circ}$ lon) GLA Fourth Order GCM has a pronounced climate drift toward a state characterized by a westerly bias in the midlatitudes of the winter hemisphere which is not nearly so evident in the lower resolution $\left(4^{\circ}\right.$ lat by $5^{\circ}$ lon) version of the model. As a result, the improvement in forecast skill brought about by the higher resolution lasts only about 5 days after which the worsened climate drift overcomes the advantages of higher numerical accuracy.

Boer et al. (1984), Sadourny (1985), Palmer et al. (1986), McFarlane (1987) and others have suggested that the westerly bias in atmospheric general circulation models could be alleviated through the parameterization of the effects of vertically propagating sub-gridscale, orographically-forced gravity waves.

Following Palmer et al. (1986), we have implemented a gravity wave drag parameterization scheme based on linear theory for gravity waves in the hydrostatic, nonrotating range. As in the scheme of Palmer et al., the surface stress due to the waves is proportional to the sub-grid-scale variance of the orography, limited by the value $(400 \mathrm{~m})^{2}$, and the stress propagates upward without change until it reaches a critical level, where it is absorbed, or until the gravity waves acquire a large amplitude and break. After the waves break, the stress is deposited according to the wave saturation hypthesis of Lindzen (1981). Our scheme imposes a Froude number constraint on the surface stress to represent the nonlinear limitations on vertically propagating gravity wave stress and employs a wavebreaking criterion which is slightly simpler than that of Palmer et al. (1986).

The effects of the parameterization scheme are immediately apparent at stratospheric levels, where most of the westerly deceleration takes place. At lower levels, however, the effect of the drag is indirect through a meridional secondary circulation generated by the Coriolis torque in response to the stratospheric deceleration. For this reason, the effect of the gravity waves becomes apparent in the troposphere only after about 5 days. This results in a reduction of the westerly bias in the Northern Hemisphere, as expected, and in an improvement in forecast skill equivalent to about 12 hours. With the parameterization, the high resolution model remains more skillful than the coarser model throughout all 10 days of the forecast.

The error reduction in the stratosphere is confined mainly to the zonally symmetric component of the flow. It does result, however, in the removal of spurious stratospheric jet streaks as long as they occur in the vicinity of mountainous regions. Error reduction is significant in both the symmetric and the nonsymmetric flow components in the middle and lower troposphere.

Improvements in the Northern Hemisphere westerlies were observed in a 31-day integration. Strong low level westerlies which penetrated into southern and western Europe were considerably weakened and shifted northward in better agreement with climatology when gravity wave drag was included in the model.

An unexpected result was observed in the Southern Hemisphere, where the control simulation exhibited a rather weak belt of low pressure at $60^{\circ} \mathrm{S}$. Gravity waves generated along the edge of the Antarctic continent in the region of surface easterlies were absorbed near the $600 \mathrm{mb}$ level and resulted in a westerly acceleration of the low level flow. This considerably improved the simulation of the roaring forties and fifties in the Southern Hemisphere.

The results presented in this study suggest that gravity wave drag has an important, positive impact on the predictive skill and the ability to simulate climate of the high resolution GLA Fourth Order Model. This gives us encouragement to continue the development of our parameterization scheme. We shall investigate the impact on forecast skill and model climatology of the following improvements to the parameterization scheme. We will Fourier filter the sub-grid-scale variance of the orography to remove spurious contributions to the wave stress from the features which are too large, such as continental slopes, or too small. Both the variance and 
its effective wave number will be computed as functions of the direction of the surface wind with the functional parameters individually predetermined for each grid point. Next, we shall incorporate into our model some of Schoeberl's (1987) findings with a steady-state WKB model concerning the level of initial wave breaking and the rate of diminution of wave amplitude due to turbulence. In addition, we shall increase the vertical resolution of the stratosphere to make it possible to compute the fluxes at $10 \mathrm{mb}$ rather than arbitrarily set them equal to zero. Finally, we shall investigate the possibility of including shear-driven gravity waves, such as those thought to be associated with the jet stream, in the model to improve the simulation of the upper level winds.

We shall also pay special attention to the improvements that were obtained for the Southern Hemisphere January climatology. These were unexpected because it was not thought that the gravity wave stress that was generated in the summer hemisphere would be strong enough to show significant impact and because they resulted in the enhancement of the westerlies. Thus we will carry out further January simulations as well as integrations for other seasons.

Finally, we plan to study the relative roles of enhanced topography and gravity wave drag and the impact of including the drag associated with the low level breaking of gravity waves in the presence of large amplitude orographic fluctuations.

\section{Acknowledgements}

We are grateful to Drs. R. Lindzen, M. Schoeberl, K. Takano and H. Tanaka for their helpful discussions concerning the development of our scheme and for their encouragement. We also wish to acknowledge the substantial contributions of Mr. P. Mullenmeister and Mr. D. Lamich for their help with the model integrations and diagnostics, Mr. R. Rosenberg for his help with the graphics, Ms. L. Rumberg and Mr. B. Sherbs for their help with the drafting and Ms. M. Wells and Mr. J. Timbs for typing the manuscript. A portion of this research was supported by NASA Grant
NSG-5077 to SUNY, Purchase.

\section{Appendix}

We expect that the upper level winds in the middle latitudes will be in quasi-geostrophic balance. This can only be true, however, if the force exerted on those winds by gravity wave stress divergence is significantly smaller than the Coriolis force exerted on the wind. That is, we expect

$$
\left|\frac{\partial}{\partial z} \rho \overline{u w}\right| \ll \rho_{u} f U_{u},
$$

where $f$ is the Coriolis parameter and the subscript $u$ refers to the values of $\rho$ and $U$ at the upper level. We assume that gravity wave stress that reaches the tropopause decreases from essentially its surface value to zero in a vertical layer of about $100 \mathrm{mb}$ which is also the approximate thickness of a model layer in the GLA Fourth Order GCM. Then using (1), we rewrite (A.1) as

$$
\frac{g \rho_{u}}{\Delta p} \rho_{s} U_{s} N_{s} k \sigma_{h}^{2} \ll \rho_{u} f U_{u},
$$

where $g$ is the acceleration of gravity, $\Delta p$ is $100 \mathrm{mb}$ or $10^{4} \mathrm{Nm}^{-2}, k=2.5 \times 10^{-5} \mathrm{~m}^{-1}$ and the subscript $s$ refers to surface values of $\rho, U$ and $N$. The requirement on $\sigma_{\bar{h}}^{2}$ is

$$
\sigma_{h}^{2} \ll \frac{f U_{u} \Delta p}{g \rho_{s} U_{s} N_{s} k} .
$$

With typical midlatitude values $f \approx 10^{-4} \mathrm{~S}^{-1}$, $U_{u} \approx 40 \mathrm{~ms}^{-1}, U_{s} \approx 10 \mathrm{~ms}^{-1}, \quad g \approx 10 \mathrm{~ms}^{-2}, \quad \rho_{s} \approx 1$ $\mathrm{kgm}^{-3}, N_{s} \approx 10^{-2} \mathrm{~s}^{-1}$, (A.3) becomes

$$
\sigma_{h}^{2} \ll 1.6 \times 10^{6} \mathrm{~m}^{2},
$$

and if we require the gravity wave drag to be an order of magnitude smaller than the Coriolis force, we have

$$
\sigma_{h}^{2} \leqq 1.6 \times 10^{5} \mathrm{~m}^{2} \equiv(400 \mathrm{~m})^{2} .
$$

\section{References}

Boer, G. J., N.A. McFarlane, R Laprise, J.D. Henderson and J.-P. Blanchet, 1984: The Canadian Climate Centre Spectral Atmospheric General Circulation Model. Atmos.-Ocean, 22, 397-429.

Chouinard, C., M. Béland and N. McFarlane, 1986 : A simple gravity wave drag parameterization 
for use in medium-range weather forecast models. Atmos.-Ocean, 24, 91-110.

Eliassen, A. and E. Palm, 1961: On the transfer of energy in stationary mountain waves. Geofys. Publ., 22, 1-23.

Helfand, H. M., J. Pfaendtner and R. Atlas, 1987 : The effect of increased horizontal resolution on GLA Fourth Order Model forecasts Short-and Medium-Range Numerical Weather Prediction (This volume), 303-315.

Kalnay, E., R. Balgovind, W. Chao, D. Edelmann, J. Pfaendtner, L. Takacs and K. Takano, 1983: Documentation of the GLAS Fourth Order General Circulation Model, Vols. 1, 2 and 3. NASA Tech. Memo. 86064, Goddard Space Flight Center, Greenbelt, MD.

Kitchen, J. and A. Dickinson, 1987: The effect of a simple parameterization of gravity wave drag in the operational forecast model. To be submitted.

Lindzen, R.S., 1981: Turbulence and stress due to gravity wave and tidal breakdown. J. Geophys. Res., 86, 9707-9714.

McFarlane, N.A., 1987: The effect of orographically excited gravity wave drag on the general circulation of the lower stratosphere and troposphere. Submitted to J. Atmos. Sci.

Miles, J.W. and H.E. Huppert, 1969: Lee waves in a stratified flow. Part 4 Perturbation aproximations. J. Fluid Mech., 35, 497-525.

Palmer, T. N., G. J. Shutts and R. Swinbank, 1986: Alleviation of a systematic westerly bias in general circulation and numerical weather prediction models through an orographic gravity wave drag parameterization. Quart. J. R. Met. Soc., 112, 1001-1039.

Peltier, W.R. and T.L. Clark, 1983: Nonlinear mountain waves in two and three spatial dimensions. Quart. J.R. Met. Soc., 109, 527-548.

Pfaendtner, J., R. Balgovind, D. Duffy, H.M. Helfand and E. Kalnay, 1985: Response of the GLA Fourth Order model to changes in horizontal resolution and terrain heights. Preprints Seventh Conf. Numerical Weather Prediction. Montreal, Amer. Meteor. Soc., 333-335.

Sadourny, R., 1985: Midlatitude systematic errors in numerical models. In GARP Spec. Rep. No. 43 : Report of the Seminar on Progress in Numerical Modelling and the Understanding of Predictability as a Result of the Global Weather Experiment. October 1984. Sigtuna, Sweden, WMO/TD-No. 33, 111, pp. 156-174.

Schoeberl, M.R., 1987: A model of stationary gravity wave breakdown with convective adjustment Submitted to J. Atmos. Sci.

Slingo, A. and D. W. Pearson, 1987: A comparison of the impact of an envelope orography and of a parameterization of orographic gravity-wave drag on model simulations. Dynamical Climato$\log y$ Technical Note No. 43, Meteorological Office, Berkshire, U. K. Submitted for publication in Quart. J.R. Met Soc.

Smith, R.E., 1979: The influence of mountains in the atmosphere. Adv. Geophys., 21, 87-230.

Tenenbaum, J., 1982: Integrated and spectral energetics of the GLAS General Circulation Model. Mon. Wea. Rev., 110, 962-980.

, 1983: Stratospheric wind errors, initial states, and forecast skill in the GLAS General Circulation Model. Mon. Wea. Rec., 111, 17361745.

, 1987: Jet stream velocity errors in general circulation models. Submitted to Mon Wea. Rev. 